DOI: https://doi.org/10.11144/Javeriana.upsy18-4.csct

\title{
Características de la supervisión clínica en las terapias conductuales: un análisis del proceso de supervisión clínica*
}

Characteristics of Supervision in Behavior Therapies: An Analysis of the Clinical Supervision Process

Recepción: 03 Octubre 2017 | Aceptación: 03 Noviembre 2019

\author{
Blanca P. Ballesteros de Valderrama ${ }^{a}$ \\ Pontificia Universidad Javeriana, Colombia \\ ORCID: http://orcid.org/0000-0003-1644-8945 \\ Amanda M. Muñoz-Martínez \\ Universidad de Los Andes, Colombia \\ ORCID: http://orcid.org/0000-0001-5558-4355 \\ Mónica Novoa-Gómez \\ Fundación Universitaria Konrad Lorenz, Colombia \\ ORCID: http://orcid.org/0000-0003-2434-6308 \\ Derkind Bazzani-Orrego \\ Pontificia Universidad Javeriana, Colombia \\ ORCID: http://orcid.org/0000-0002-6561-157X \\ Natalia E. Brandwayn-Briceño \\ Pontificia Universidad Javeriana, Colombia \\ ORCID: http://orcid.org/0000-0001-5687-1778 \\ R. Andrés LASSO-BÁEZ \\ Fundación Universitaria Konrad Lorenz, Colombia \\ ORCID: http://orcid.org/0000-0002-0529-8323 \\ Mónica Pachón-Basallo \\ Universidad de Sevilla, España \\ ORCID: http://orcid.org/0000-0003-2339-2708 \\ David Restrepo-VÉlez \\ Pontificia Universidad Javeriana, Colombia \\ ORCID: http://orcid.org/0000-0001-7723-6503
}

a Autor de correspondencia. Correo electrónico: bpballesteros@gmail.com

Para citar este artículo: Ballesteros de Valderrama, B. P., Muñoz-Martínez, A. M., Novoa-Gómez, M., BazzaniOrrego, D., Brandwayn-Briceño, N. E., Lasso-Báez, R. A., ... Restrepo-Vélez, D. (2019). Características de la supervisión clínica en las terapias conductuales: un análisis del proceso de supervisión clínica. Universitas Psychologica, 18(4), 1-14. https://doi.org/10.11144/ Javeriana.upsy18-4.csct

\section{RESUMEN}

Se presentan las características de la supervisión clínica conductual abstraídas de un análisis de tipo etnográfico de entrevistas realizadas a 18 supervisores de la primera, segunda y tercera generación de terapias conductuales. Los entrevistados contaban con experiencia de 17 años en promedio $(D E=9.85)$ como clínicos y 16 años en promedio $(D E=10.51)$ como supervisores. Se utilizó el software Altlas.ti 6.2 para analizar y relacionar las características centrales de la supervisión. Los resultados mostraron la supervisión conductual como categoría central con dos subcategorías axiales: factores que afectan la supervisión y proceso de supervisión. Los procedimientos de enseñanza con mayor fundamentación fueron: la formulación de caso, la discusión teórica, la retroalimentación, el modelamiento y el moldeamiento. Se concluye que la supervisión clínica se conceptualiza como un proceso y no como un resultado, y 
se discuten las implicaciones para los programas psicología clínica en la formación de futuros terapeutas.

Palabras clave

supervisión clínica; terapias conductuales; formación clínica; proceso de supervisión; educación informal.

\begin{abstract}
The characteristics of behaviorally based clinical supervision are presented. Eighteen open-ended interviews were conducted to supervisors from first, second, and thirdwave behavioral therapies. These were analyzed based on an ethnographic approach. Participants reported an average of 17 -years $(S D=9.85)$ practicing as clinicians and 16-years $(S D=10.51)$ as supervisors. The Altlas.ti 6.2 software was employed to analyze and synthesize the central processes embedded in supervision. Results found behavioral oriented supervision as the core category which encloses the following two axial subcategories: factors influencing supervision and supervision process. Case conceptualization, theoretical discussion, verbal feedback, shaping, and modeling were the most utilized teaching methods by the experts interviewed. We concluded that clinical supervision would be conceptualized as a process rather than an outcome. We discussed implications for clinical psychology programs when training future clinicians.

Keywords

clinical supervision; behavior therapies; clinical training; supervision process; continue education.
\end{abstract}

La relevancia disciplinar y social de la supervisión clínica ha llevado a psicólogos clínicos e investigadores del área a interesarse por el desarrollo de guías que regulen y mejoren los procesos de supervisión (Gazzola, De Stefano, Thériault, \& Audet, 2014). Disciplinas encargadas de mejorar la salud mental ( $p$. ej., psiquiatría) en países europeos, americanos y oceánicos, han impulsado el diseño de parámetros que organicen las prácticas en la supervisión clínica (American Psychological Association, 2015; Bernard, 2006; Weerasekera, 2013). Por ejemplo, en Chile y Argentina el ejercicio de supervisión debe ser acreditado por una comisión de acreditación clínica. En los últimos años, se han escrito diversos libros y artículos que han sustentado la importancia de establecer guías de supervisión y evaluar su utilidad en la formación de terapeutas (Milne, 2018; Watkins \& Milne, 2014).
Watkins (2014) identifica cuatro temas claves relacionados con la supervisión clínica que necesitan ser investigados y parametrizados: la medición, la diversidad, la investigación y el entrenamiento y la práctica en supervisión. La mayoría de investigadores en esta área resaltan la importancia de la formación de supervisores competentes y de identificar las mejores prácticas para estructurar experiencias de entrenamiento, y señalan la falta de investigación que muestre el impacto de la supervisión clínica.

Aunque desde la última década del siglo veinte, la investigación de los efectos de la supervisión clínica ha crecido con el diseño de la Batería de Investigación en Supervisión (auspiciada por la British Association for Counselling and Psychotherapy), los trabajos para establecer una agenda específica sobre la supervisión presentan limitaciones de tipo metodológico que requieren trabajo investigativo. Lo anterior hace importante recabar evidencia que apoye o refute el uso de estrategias y técnicas específicas en la supervisión clínica, y así determinar sus elementos fundamentales (p. ej., Bernard \& Goodyear, 2014; Ellis et al., 2014; Falender \& Shafranske, 2017; Goodyear \& Rodolfa, 2012; Newman, 2013; Watkins, 2014).

Para la psicología conductual, la supervisión clínica es un reto, dadas las diferencias a nivel teórico y epistemológico entre las diversas propuestas terapéuticas de este modelo. Hayes (2004) plantea que la terapia conductual ha pasado por tres etapas desde su configuración: la primera generación de terapias conductuales se fundamenta en los principios analítico-conductuales propuestos por B. F. Skinner, y requieren que el terapeuta maneje sistemáticamente el contexto de los individuos para conseguir cambios en su conducta. La segunda generación mantiene la importancia de los factores contextuales, no obstante, introduce como factores causantes de la conducta los eventos de tipo cognitivo y emocional, por lo que asume un modelo internalista y dualista de la conducta. Finalmente, la tercera generación retorna a una visión monista del comportamiento, sin ignorar el papel de la 
cognición y la emoción en el control de la conducta humana. Esta aproximación plantea que el comportamiento de los individuos es influido tanto por el contexto físico como por el contexto socio-verbal, y debido a ello los terapeutas deben ser entrenados en evaluar e intervenir los diversos tipos de contextos para producir cambios terapéuticos.

Aunque las diferentes terapias de conducta tienen como objeto central la modificación del comportamiento del consultante, las acciones terapéuticas para conseguir dicho objetivo divergen entre ellas (Bastidas-Bilbao \& Velásquez, 2016). En la actualidad, las terapias de segunda generación, especialmente la Terapia Cognitivo Conductual (CBT, por sus siglas en inglés) han desarrollado diferentes modelos de supervisión. Johnston y Milne (2012) mencionan que la supervisión clínica en la CBT se fundamenta en los mismos procesos y métodos que el proceso terapéutico, por lo que se espera que el supervisor clínico establezca objetivos de supervisión al inicio de las sesiones, señale la importancia de la colaboración dentro del proceso y anime al supervisado a seguir un camino de descubrimiento dentro de su formación. En las terapias de primera y tercera generación, se ha planteado que los principios conductuales que se siguen para modificar el comportamiento deberían ser aplicados de manera similar por los supervisores cuando enseñan habilidades clínicas (Callaghan, 2006). Follette y Callaghan (1995) plantean que el entrenamiento de psicólogos clínicos conductuales requiere moldear en el supervisado comportamiento efectivo con el supervisor en la sesión de supervisión, así como realizar retroalimentación en vivo de las conductas del terapeuta en sesión. Para estos autores, es importante que los supervisores reduzcan el control instruccional en el entrenamiento de los clínicos, dado que este tipo de estrategias pueden limitar la variabilidad conductual de los supervisados, afectando su efectividad en circunstancias terapéuticas novedosas. En esta línea, es posible encontrar estudios que analizan las variables de la interacción ( Novoa-Gómez et al., 2019).
Aunque diferentes guías indican la conducta esperada de los supervisores conductuales con base en los principios conductuales, es importante mencionar la carencia de estudios que describan las prácticas y los elementos en la supervisión clínica conductual con base en la experiencia de los actores involucrados en dicho proceso. En general, los autores muestran que la relación entre las habilidades terapéuticas, como producto, y los procesos implicados en el desarrollo o la formación de estas, parecen ser independientes de los enfoques teóricos adoptados por los psicólogos clínicos (Cruz, 2009; Hernández, 2007; Moreira, Sabola, Beco, \& Soares 1995; Nel, Pezzolesi, \& Stott, 2012; O'Donovan, Halford, \& Walters, 2011; Shaw, 2013), sin mencionar los elementos prácticos que favorecen el aprendizaje de dichas habilidades. Así mismo, es importante investigar las características de los supervisores como actores fundamentales dentro del proceso de aprendizaje de las habilidades clínicas, como señala Barnett (2014).

La presente investigación buscó caracterizar la supervisión clínica de terapeutas conductuales de primera, segunda y tercera generación, estableciendo los factores involucrados en ellas y las características del proceso en general. A diferencia de otros estudios, como el llevado a cabo por Johnston y Milne (2012) en el que se entrevistó a supervisados a partir de los parámetros de la teoría fundamentada, en este estudio se adoptó una metodología de tipo etnográfico, que permitió entender el proceso de supervisión a partir de la experiencia de los supervisores como actores centrales en el proceso de supervisión.

\section{Método}

\section{Tipo de investigación}

Investigación de tipo etnográfico para describir y analizar las prácticas, significados y conocimientos de un fenómeno con base en la perspectiva de sus actores (Salgado, 2007; Taylor \& Bogdan, 1987). Esta metodología 
permitió identificar las regularidades en el ambiente que influyen en la supervisión clínica, así como agrupar las estrategias utilizadas en la supervisión, para categorizar la actividad en dicho contexto, identificar la estructura y los procesos involucrados en la supervisión con una aproximación inductiva en la que el punto de vista de los participantes permitió construir una comprensión colectiva de la supervisión conductual. Con base en el método comparativo constante, se contrastaron los datos desde que inició la recolección de información, se identificaron similitudes y diferencias entre los expertos entrevistados, y se establecieron patrones de conducta o sucesos que permitieron la formulación de hipótesis que relacionaban los elementos claves de la supervisión clínica conductual (Strauss \& Corbin, 2002).

\section{Participantes}

Se llevó a cabo un muestreo teórico caracterizado por recoger información y generar teoría con una muestra inicial con base en el método comparativo constante. El muestreo fue saturado con 18 entrevistas. Los supervisores clínicos entrevistados eran expertos en terapias conductuales y cognitivo-conductuales, con suficiencia académica, experiencia y certificación en una o varias de las siguientes aproximaciones terapéuticas: Activación Conductual, Análisis Conductual Aplicado (ABA, por sus siglas en inglés), Psicoterapia Analítico Funcional (FAP, por sus siglas en inglés), Terapia de Aceptación y Compromiso (ACT, por sus siglas en inglés), Terapia Cognitivo-Conductual (CBT, por sus siglas en inglés) y Terapia Dialéctico Conductual (DBT, por sus siglas en inglés). La aproximación terapéutica, el nivel educativo y lugares de origen de los entrevistados se presentan en la Figura 1.
Figura 1

Distribución y número de participantes por aproximación terapéutica.

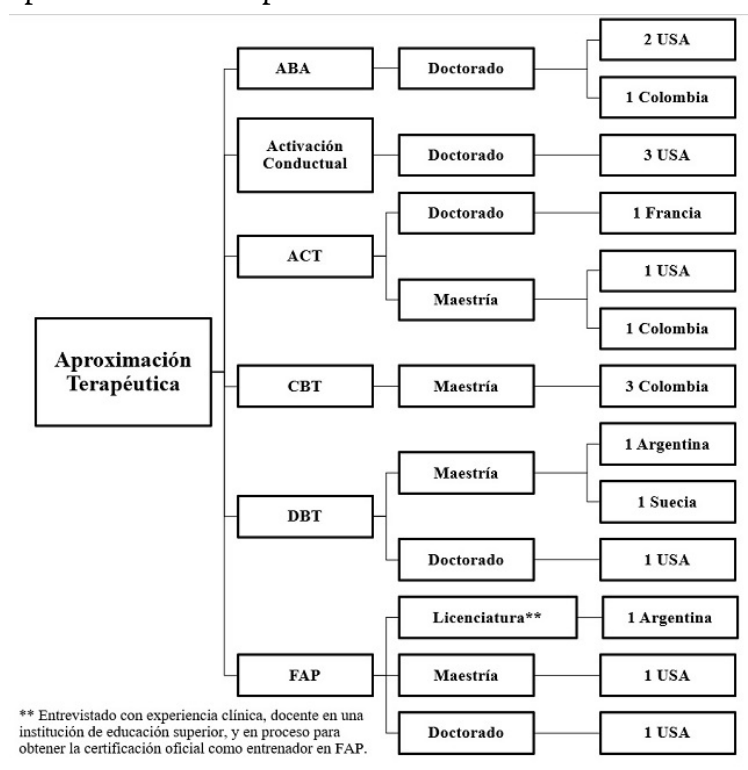

Algunos de los participantes se encontraban certificados o tenían experiencia en más de una de las aproximaciones terapéuticas de interés, sin embargo, para evitar traslapar la información se solicitó explícitamente que al responder las preguntas hicieran referencia de manera específica a la terapia por la cual se habían contactado para la investigación. Todos los participantes contaban con experiencia como psicólogos clínicos entre tres y 38 años $(M=17$; $D E=9.85)$ y como supervisores clínicos entre tres y 25 años $(M=16 ; D E=10.51)$.

\section{Instrumentos}

\section{Entrevista semiestructurada}

Este tipo de entrevista permite indagar de manera flexible los aspectos pertinentes al área de estudio, de manera que el entrevistado puede obtener información sobre aspectos no especificados explícitamente en el formato de entrevista, pero que pueden ser importantes para el análisis. Este tipo de entrevista facilita la recolección de información detallada y completa, ahondando en el fenómeno de interés. 
La entrevista contó con 27 preguntas en tres categorías sugeridas por la literatura en supervisión clínica: (a) características del entrenamiento que habían recibido los entrevistados para convertirse en supervisores, (b) actividades dentro de la supervisión y (c) factores que afectaban la supervisión realizada. Las preguntas permitieron explorar las experiencias y dinámicas de los expertos durante su entrenamiento clínico y el comportamiento actual con sus supervisados. El formato guía de la entrevista fue evaluada por expertos en psicología clínica conductual y terapias cognitivo-conductuales en forma y contenido, y se realizaron tres entrevistas iniciales con supervisores clínicos, las cuales permitieron abstraer aquellas preguntas que brindaban más información en dirección a los objetivos planteados.

\section{Procedimiento}

Inicialmente, se establecieron las preguntas guía para llevar a cabo la entrevista a profundidad. Posteriormente, los procedimientos fueron evaluados por el Comité de Investigación y Ética institucional, garantizando el cumplimiento de los estándares de la Ley 1090 del 2006 y el respectivo Manual Deontológico y Bioético (COLPSIC, 2009) y el Acuerdo de Helsinki (APA, 2002).

Se determinó entrevistar a tres supervisores por cada una de las aproximaciones terapéuticas dentro del modelo conductual y evaluar posteriormente la saturación de los códigos, y así reevaluar la muestra. Los participantes fueron contactados de manera individual a través de correos electrónicos o comunicaciones personales. En el momento de contacto, se les informó el objetivo de la investigación, se solicitó consentimiento informado de tipo verbal para garantizar el uso ético de sus datos personales y la divulgación de la información resultante del análisis de los datos. Los participantes también fueron instados a realizar preguntas para aclarar elementos que consideraran importantes con respecto al estudio. Las entrevistas planeadas generaron un nivel óptimo de saturación para las categorías analizadas, por lo que no fue necesario contactar otros expertos. Las entrevistas llevadas a cabo en el país de origen de los investigadores se realizaron personalmente, mientras que los participantes en otros países fueron entrevistados a través de videollamadas. Finalmente, estas fueron transcritas y traducidas (cuando fue necesario) para su análisis.

\section{Análisis de datos}

Las entrevistas fueron organizadas en unidades hermenéuticas en el software Atlas ti 6.2. Se realizó un análisis de contenido de la información recolectada que permitió identificar las diferencias y similitudes de las narrativas de cada uno de los participantes y establecer relaciones jerárquicas entre categorías, lo que facilitó agrupar y establecer estructuras de los verbatims/narrativas para comprender el proceso y la práctica de la supervisión conductual.

En el análisis de contenido, inicialmente se realizó una codificación abierta línea a línea de lo transcrito, lo que permitió la abstracción de códigos sustantivos e in vivo, así como memos sobre las consistencias e inconsistencias entre los entrevistados, al analizar las entrevistas. En este nivel de análisis, se llevó a cabo la comparación constante de los datos, lo que posibilitó la saturación de las categorías y así proceder a la codificación axial. Posteriormente, se llevó a cabo la codificación axial de los códigos sustantivos derivados del primer tipo de codificación, con el fin de establecer relaciones entre estos códigos y los códigos teóricos (establecidos a partir de los memos), y así alejar los datos de simples descripciones implicadas en las transcripciones. Finalmente, se completó la codificación selectiva con la que se generaron las categorías centrales o núcleo en las que se agruparon las categorías previamente analizadas y se establecieron las estructuras de la supervisión clínica conductual. Así mismo, se llevó a cabo un análisis descriptivo de las frecuencias de las palabras representativas dentro de las categorías identificadas, haciendo 
uso de la herramienta de análisis de datos Microsoft Excel.

\section{Resultados}

El análisis de contenido de las narrativas derivadas de las entrevistas se encuentra representado en redes conceptuales (estructurales) y gráficos de frecuencia. Tras el análisis de las narrativas se derivó la familia Supervisión Conductual (Figura 2), que enmarca dos categorías principales: Factores que afectan la supervisión y Proceso de supervisión. Estas dos categorías muestran que, en las terapias conductuales, los supervisores guían el proceso de supervisión teniendo en cuenta los elementos del contexto de los terapeutas y de ellos como supervisores que pueden afectar el aprendizaje, con el fin de garantizar condiciones óptimas para este. Además, se observa que los supervisores establecen un plan que orienta las actividades de enseñanza con estrategias propias de su modelo, con el fin de fomentar el aprendizaje de elementos teóricos y prácticos. En la categoría Factores que afectan la supervisión, se encuentran los factores estructurales, factores personales e interpersonales, mientras que en la de Proceso de supervisión, se encuentran los objetivos de formación de la supervisión y procedimientos de enseñanza en la supervisión.
Figura 2

Categoría nuclear de la supervisión conductual.

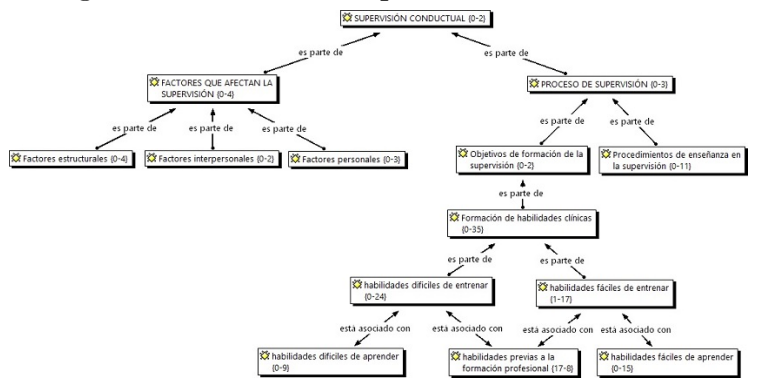

Los valores numéricos en las redes estructurales hacen referencia al número de códigos que se encuentran dentro de una misma categoría y la cantidad de relaciones entre categorías, estos dos elementos se encuentran entre corchetes; por ejemplo, en la categoría "habilidades previas a la formación profesional" $\{17-8\}, 17$ códigos asociados a la categoría y 8 , a la relación con otras categorías. Fuente: elaboración propia.

Los factores estructurales incluyen elementos de organización del espacio de supervisión, donde destacan las modalidades de supervisión y el tiempo disponible para esta. La supervisión grupal fue la modalidad de mayor fundamentación $\{15\}$, importante para los supervisados porque les permite tomar perspectiva de los otros terapeutas, desarrollar empatía y aprender la habilidad de "metaobservación" del proceso de supervisión de manera directa: "Lo que me gustó fue que brinda la perspectiva de otros supervisados, por lo que puedes compartir puntos de vista". Otra modalidad con una menor fundamentación fue la individual $\{5-1\}$. La supervisión en vivo $\{13-1\}$ permite un acompañamiento cercano de los supervisores a la consulta de sus estudiantes, y constituye la posibilidad de aprender las habilidades clínicas a través del moldeamiento y modelamiento, como lo mencionó uno de los entrevistados "el estudiante interactúa con consultantes, pero siempre siendo acompañado y observado por su supervisor". Cabe mencionar que, aunque las supervisiones presenciales ( $\mathrm{p}$. ej., en vivo, grupal) son las modalidades más frecuentes, el uso de supervisiones virtuales $\{5-1\}$ fue referida como una modalidad utilizada por algunos de los entrevistados, especialmente 
cuando los estudiantes buscan aprender ciertas terapias a profundidad.

En cuanto al tiempo disponible para la supervisión, la experiencia del supervisado fue un criterio determinante del número de horas necesarias para una supervisión eficaz, siendo mayor en los primeros estadios de entrenamiento y disminuyendo conforme aumentaba la experiencia: "un estudiante inicial, que comienza a ver pacientes debería tener una hora de supervisión por cada hora con el cliente que tenga". De acuerdo con los entrevistados, el número de consultantes y la cantidad de horas de atención también son elementos importantes para determinar el tiempo invertido en la supervisión.

Por otro lado, los espacios de supervisión se organizaban en función de una perspectiva ideográfica, donde las particularidades individuales y las necesidades de cada estudiante eran consideradas para el proceso de supervisión.

Respecto a los factores interpersonales, la relación supervisado-supervisor fue una categoría relevante dentro del proceso de supervisión $\{27-1\}$. Varias narrativas señalaban el impacto de una buena relación durante el entrenamiento clínico; por ejemplo, "si no hay un buen vínculo, se dificulta el aprendizaje del supervisado y por ende eso va a repercutir en el trabajo con sus pacientes". Para los entrevistados, la relación con sus supervisados es un elemento fundamental en la consecución de resultados positivos en la enseñanza de habilidades y los procedimientos terapéuticos. Algunos señalaron comportamientos por parte de los supervisados que afectaban la consolidación de una relación supervisado-supervisor efectiva; por ejemplo, uno de los supervisores señalaba "a veces quieren verse [los supervisados] bien o ser muy complacientes con el supervisor (...) solo reportando las cosas buenas". Esto muestra la importancia de analizar la función de la conducta del supervisado dentro de la supervisión, acordar objetivos mutuos, y establecer una comunicación clara entre el supervisor y el supervisado.

En la categoría de factores personales (Figura 2), se encontró que las características del supervisor y del supervisado pueden influir en el proceso de supervisión. En las características del supervisado, los entrevistados incluyeron: el nivel de formación $\{67-1\}$, las habilidades previas a la formación profesional $\{17-9\}$ y los patrones de evitación $\{8-1\}$.

Respecto a las características del supervisor (Figura 3), los entrevistados identificaron características personales que podrían interferir con la supervisión. Algunos de los entrevistados reconocieron que factores de su historia personal y profesional podían influir en su papel como supervisor. Uno de los entrevistados mencionó: "hay situaciones en las cuales yo no puedo supervisar, tengo problemas personales con ciertos temas".

\section{Figura 3}

Factores personales y características del supervisor, en donde se incluye su formación como clínico y como supervisor.

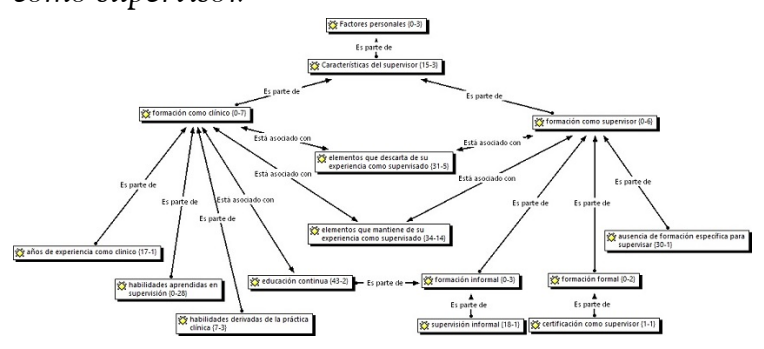

En la categoría de formación como supervisor (Figura 3), los participantes reportaron no haber recibido formación específica que los capacitara como supervisores durante su educación formal $\{30-1\}$, a excepción de un participante que tiene una certificación como supervisor $\{1-1\}$ de una universidad de Estados Unidos. A pesar de esta ausencia de formación formal para supervisar, durante el entrenamiento para convertirse en psicólogos clínicos o analistas conductuales aplicados, adquirieron habilidades que les permitían realizar su labor como supervisores. Algunos entrevistados mencionaron realizar supervisión informal $\{18-1\}$ con el fin de manejar situaciones complejas en la supervisión o el contexto clínico. Por otro lado, los entrevistados refirieron participar en cursos de educación continua $\{43-2\}$ con el propósito de actualizar su conocimiento en el área y desarrollar habilidades profesionales, incluidas las de supervisión. La 
mayoría de entrevistados reportaron que las habilidades aprendidas para supervisar fueron derivadas de su entrenamiento como psicólogos clínicos, constituyendo una de las categorías principales en su formación. En la Figura 4 , se muestran las habilidades aprendidas en supervisión, algunas de ellas con una mayor fundamentación, como la formulación de caso $\{41\}$, la retroalimentación $\{32\}$, la toma de riesgos $\{13\}$ y las habilidades de evaluación $\{11\}$. Adicionalmente, señalaron que las habilidades clínicas no solamente las aprendieron en la supervisión, sino a través de la experiencia clínica, en la cual fueron moldeadas habilidades como la empatía $\{22\}$ y la lectura de contexto $\{7\}$.

\section{Figura 4}

Habilidades aprendidas en supervisión clínica.

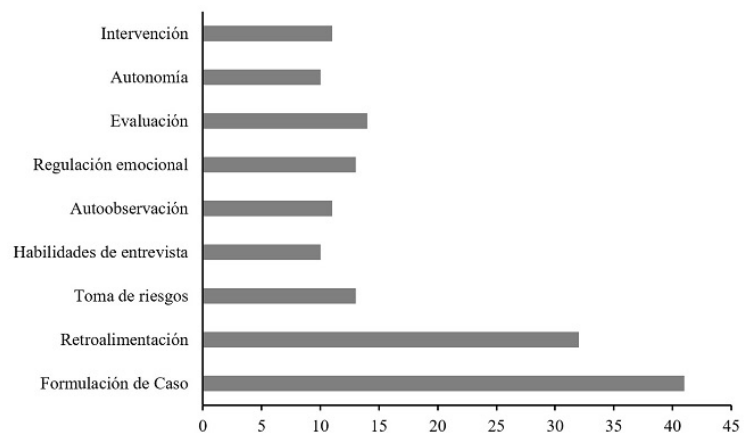

La otra gran categoría que se desprende de la familia Supervisión Conductual es la de Proceso de supervisión, que conglomera otras subcategorías: objetivos de formación de la supervisión y procedimientos de enseñanza en la supervisión (Figura 2). Dentro de la primera de estas subcategorías, se consolidó la categoría de formación de habilidades clínicas $\{0-35\}$ (Figura 2). Esta categoría incluye las habilidades difíciles y fáciles de entrenar, las habilidades fáciles y difíciles de aprender y las habilidades previas a la formación profesional (Figura 2).

Con respecto a las habilidades clínicas que los supervisores enseñan en supervisión, se incluyeron habilidades fáciles y difíciles de entrenar (Figuras 2). Como se observa en la Figura 5, la empatía y la intervención pueden ser fáciles de entrenar para algunos supervisores, pero no para otros. Es posible que en función de las características personales del supervisado, el nivel de dificultad cambie. Es de destacar que algunos entrevistados señalaron que ninguna habilidad es más fácil o difícil de aprender o de entrenar per se, de hecho, el grado de dificultad de las habilidades se relacionó con la categoría de habilidades previas a la formación profesional. Se encontró que habilidades difíciles de aprender también resultaban difíciles de entrenar con sus supervisados.

\section{Figura 5}

Habilidades fáciles y difíciles de aprender y de entrenar en el proceso de supervisión clínica.

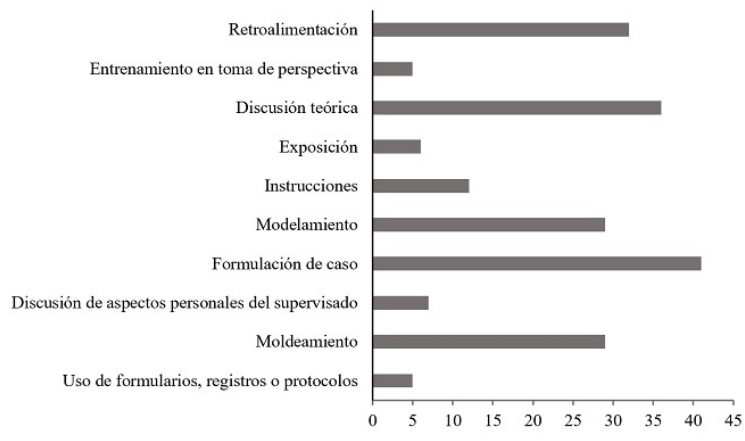

En cuanto a la categoría Procedimientos de enseñanza en la supervisión, en la Figura 6 se muestra la fundamentación de los mismos. Se observó que la formulación de caso $\{41-7\}$ y la retroalimentación $\{29-8\}$ son tanto habilidades que se aprenden y se enseñan en la supervisión como procedimientos para la enseñanza de habilidades. Otros procedimientos de enseñanza con una fundamentación notable fueron la discusión teórica $\{36-2\}$, el moldeamiento $\{29-8\}$ y el modelamiento $\{29-5\}$; dentro de estos dos últimos, el ver videos $\{35-2\}$ y el juego de roles $\{20-2\}$ fueron dos de las actividades utilizadas para la enseñanza de habilidades clínicas con mayor fundamentación. 
Figura 6

Procedimientos de enseñanza en la supervisión.

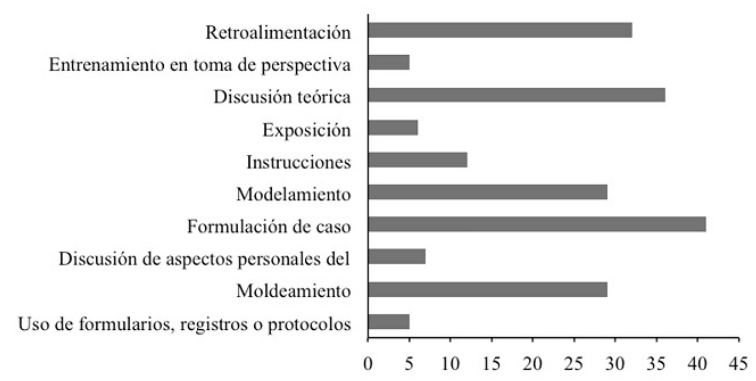

\section{Discusión}

Este estudio cumplió el objetivo de caracterizar la supervisión conductual con base en la perspectiva de los supervisores clínicos como agentes centrales en la formación de psicoterapeutas. La metodología utilizada permitió analizar las diferentes generaciones de la terapia conductual más allá de lo que la literatura supone que debe ser y de lo que se esperaría, para tener una aproximación a la supervisión desde la perspectiva de quienes están directamente vinculados en la práctica desde una óptica naturalista.

El análisis de las entrevistas mostró que para los supervisores la Supervisión Conductual como categoría central de análisis, se relaciona con dos aspectos fundamentales: (a) los factores que afectan la supervisión y (b) el proceso de supervisión como tal. Esto muestra que la supervisión conductual está anidada en una visión contextualista, entendiéndola como un proceso que implica acciones específicas dentro del contenido de la interacción terapeutasupervisor, que a su vez se encuentran influidas por factores contextuales.

El conceptualizar la supervisión como un proceso y no como un producto conlleva a que las actividades propias de la interacción supervisorsupervisado se vayan configurando a través del tiempo, y aunque tiene como fin la formación de psicoterapeutas hábiles, el resultado no es el criterio definitorio, coincidiendo con elementos que han sido desarrollados por los trabajos de Milne (2018). Adicionalmente, la supervisión como proceso también implica que se encuentre en función de un conjunto de variables históricas y contextuales, las cuales se relacionan con la experiencia previa tanto del supervisor como del supervisado, e incluye el desarrollo de habilidades personales y terapéuticas del supervisado. Respecto al desarrollo de estas habilidades personales y terapéuticas es necesario mencionar tres elementos: primero, el entrenamiento en psicoterapia ha de enfocarse en habilidades técnicas como la entrevista, su estructura y la práctica autorreflexiva; segundo, el entrenamiento ha de incluir habilidades no técnicas dentro de las que se puede mencionar, la empatía y su influencia en la relación terapeutapaciente (Alberts \& Edelstein, 1990; Ducharme, Knudsen, \& Roman, 2008; Guest \& Beutler, 1988) y tercero, ha de incluir habilidades discretas como la comunicación facilitadora, comportamientos atentos y evaluación, como lo habían señalado Bootzin y Ruggill (1988), y que ha sido recientemente evaluado también en la investigación de Novoa-Gómez et al. (2019). En esta misma dirección, los resultados también mostraron el uso de diferentes estrategias para la formación de habilidades clínicas, lo que concuerda con los mismos autores, quienes refirieron que el entrenamiento clínico es multidimensional, ya que los supervisados deben aprender métodos y procesos de intervención, formular sus casos clínicos integrando información proveniente de diversas fuentes de retroalimentación y desarrollar habilidades relacionales para la alianza terapéutica, con el fin de conseguir un proceso de supervisión exitoso y formativo (Newman, 2013).

Sumado a ello, se observó que los métodos utilizados por los entrevistados se encontraban directamente relacionados con los marcos conceptuales y metodológicos de las diferentes generaciones de la terapia conductual (p. ej., moldeamiento, modelamiento, instrucciones). Esto permite ver que para los entrevistados la coherencia entre la práctica clínica y lo establecido teóricamente es central, tema que ha sido discutido como un aspecto crítico y pobremente desarrollado en la investigación 
sobre formación de terapeutas (Barraca, 2009; Cruz, 2009; Hernández, 2007; Moreira et al., 1995; Nel et al., 2012; O'Donovan et al., 2011; Shaw, 2013).

Cabe señalar que los resultados muestran que algunos de los supervisores que se afiliaban a las terapias de tercera generación habían hecho una transición teórica y metodológica, en coherencia con lo formulado por Siddiqui (2012); varios de los supervisores de terapias de tercera generación entrevistados habían modificado aspectos esenciales en sus prácticas, incluyendo elementos de aceptación, mindfulness y toma de perspectiva, dentro de sus entrenamientos. Al respecto, vale mencionar que no todos los supervisores de tercera generación entrevistados hicieron una transición completa de una generación a otra, pues aún usaban estrategias de la primera y la segunda. Esto último podría tener implicaciones de tipo conceptual que sería importante abordar en otros estudios, ya que los terapeutas de tercera generación adoptan una perspectiva contextual no dualista del lenguaje y el pensamiento, que dista de los postulados teóricos de los terapeutas de segunda generación, como lo señala Hayes (2004).

Aunque este estudio muestra que para los supervisores es importante entrenar a los terapeutas en conceptualizar e intervenir sus casos usando estrategias coherentes con las teorías conductuales en general (p. ej., analíticoconductual, cognitivo-conductual, contextualfuncional), no fue posible determinar si las estrategias utilizadas eran coherentes con una perspectiva exclusiva o si estos transitaban entre teorías dentro de la supervisión, lo que podría llevar a un eclecticismo teórico en el entrenamiento de los terapeutas. Por lo tanto, es importante explorar con mayor precisión el tipo de relación entre la teoría y la práctica en la supervisión conductual, y si ello afecta la efectividad del entrenamiento y de la atención clínica. Además, este estudio permitió identificar las estrategias utilizadas para enseñar las habilidades clínicas en la supervisión clínica, lo cual ha sido frecuentemente señalado como una limitación en este campo de estudio (NovoaGómez, Córdoba-Salgado, 2019; Watkins, 2014).
Sin embargo, no fue posible establecer cuáles de las estrategias utilizadas son más útiles en el proceso de supervisión; para lograr este nivel de especificidad se recomienda llevar a cabo estudios experimentales que incorporen condiciones de control con el fin de contrastar los efectos del entrenamiento, usando las diferentes herramientas identificadas en este estudio.

La presente investigación llama la atención sobre el papel de la formulación del caso clínico como habilidad que se debe entrenar en la supervisión y como procedimiento de enseñanza, siendo la categoría con mayor número de verbatims tras el análisis de los datos. Esto se relaciona con los resultados del estudio de Muñoz-Martínez y Novoa-Gómez (2012), los cuales mostraron el valor de la conceptualización de caso para los terapeutas conductuales en términos de la elección de intervenciones efectivas para abordar los casos clínicos. Otros estudios también han señalado la importancia de la formulación en la conceptualización en diversos problemas clínicos (Caycedo, NovoaGómez, \& Ballesteros, 2008; Muñoz-Martínez \& Novoa-Gómez, 2011; Pulido \& Novoa-Gómez, 2014).

En cuanto a la relación supervisorsupervisado, el número de verbatims relacionados con este aspecto $\{27-1\}$, muestra la importancia de esta interacción. El análisis la evidenció como un elemento sensible del proceso, dado que puede mejorar la actuación de los terapeutas dentro de la sesión o deteriorarla. Como lo señala Ladany (2014), esto puede llevar a incompetencia pedagógica, por lo que es importante que los supervisores implementen métodos para tener mayor información de lo sucedido en la sesión, como videos o incluso retroalimentación en vivo (ver también Follette $\&$ Callaghan, 1995).

Los resultados son consistentes con la literatura revisada en cuanto a la relación entre las habilidades terapéuticas como producto y los procesos en la formación de estas en la supervisión, independiente de los enfoques teóricos (Rodolfa et al., 2013). Los entrevistados indicaron que cada supervisado tiene habilidades previas a la formación profesional, las cuales 
eran más fáciles de incrementar y moldear en las circunstancias terapéuticas, ya que hacían parte de su repertorio "natural". Esta misma lógica opera respecto a las habilidades difíciles de aprender y entrenar, ya que, si el supervisado no tiene en su repertorio ciertas habilidades, entrenarlas requiere mayor tiempo y práctica, es decir, un mayor esfuerzo en su enseñanza. Esta clasificación de las habilidades en términos de su facilidad o dificultad vale la pena aclararla en futuras investigaciones, ya que, para algunos entrevistados, no hubo habilidades fáciles $\mathrm{o}$ difíciles de entrenar.

Respecto a las fuentes informales de supervisión, los resultados son consistentes con lo mencionado por Farber y Hazanov (2014), quienes en un sondeo realizado con 146 estudiantes encontraron que, con frecuencia, aparte de las consultas a su supervisor asignado, recurrían a colegas, a su propio psicoterapeuta y a otras personas significativas, con el propósito de estar más seguros de sus actuaciones clínicas. Es importante que dentro de estos procesos se implementen consentimientos informados de manera formal con los consultantes, donde se declare que su información sería compartida con otro terapeuta.

Los hallazgos muestran la importancia de contar en los programas de psicología clínica conductual con entrenamiento directo y específico en supervisión clínica (solo uno de los entrevistados reportó haber recibido ese tipo de formación), pues la mayor parte de programas carecen de ello, llevando a que los psicólogos clínicos recurran a otros contextos que les brinden herramientas al respecto (buscan entrenamiento fuera de las aulas de clase y de tipo virtual), por lo que el contacto directo y extendido entre supervisor y supervisado ha disminuido. Resulta paradójico que, aunque diversos autores como Watkins (2014) han llamado la atención sobre incluir esta línea de formación como una modalidad de educación superior, el avance en este aspecto es poco. Como señala este autor, se requiere mejorar las condiciones en la formación de supervisores, para lograr que esos sean competentes y hábiles en la identificación de las mejores prácticas en los casos. Al respecto, los resultados también apoyan lo mencionado por Barnett (2014) sobre los supervisores competentes y comprometidos, quienes disfrutan su trabajo y lo integran con su identidad profesional y sus valores. Lo anterior parece común a la supervisión en diversos enfoques, como puede verse en Cruz (2009), Hernández (2007), Moreira et al. (1995), Nel et al. (2012), O'Donovan et al. (2011) y Shaw (2013).

En resumen, este estudio mostró que los supervisores implementan distintos procedimientos de enseñanza que se podrían complementar entre sí. Este trabajo sería importante para unificar prácticas útiles de enseñanza en las terapias conductuales y para implementar dentro de un programa de formación para supervisores. Vale aclarar que la inquietud por encontrar prácticas comunes también existe en la formación psiquiátrica, como lo describe Weerasekera (2013) en su estudio, y su llamado por un consenso internacional sobre guías de supervisión en la formación.

\section{Agradecimientos}

Esta investigación fue financiada por la Pontificia Universidad Javeriana mediante convocatoria 012, apoyo interno semilleros 2013, Id Proyecto: 005952 y fue realizada entre 2014 y 2015.

\section{Referencias}

Alberts, G., \& Edelstein, B. (1990). Therapist training: A critical review of skill training studies. Clinical Psychology Review, 10, 497-511. https://doi.org/10.1016/0272-735 8(90)90094-Q

American Psychological Association. (2002, amended 2010). Ethical principles of psychologists and code of conduct. Recuperado de http://apa.org/ethics/code/i ndex.aspx

American Psychological Association. (2015). Guidelines for clinical supervision in health service psychology. The American 
Psychologist, 70, 33-46. https://doi.org/10.10 37/a0038112

Bastidas-Bilbao, H., \& Velásquez, A. M. (2016). Modelo lógico de la supervisión como actividad educativa para la formación en psicología clínica: concepciones de supervisores clínicos. Avances en Psicología Latinoamericana, 34(2), 293-314. https://do i.org/10.12804/apl34.2.2016.06

Barnett, J. E. (2014). Introduction: The (hopefully) essential primer on clinical supervision. Journal of Clinical Psychology: In Session, 70, 1023-1029. https://doi.org/10.1 $002 /$ jclp.22123

Barraca, J. (2009). Habilidades clínicas en la terapia conductual de tercera generación. Clínica y Salud, 20 (2), 109-117. Recuperado de http://scielo.isciii.es/scielo.php?script $=$ s ci_arttext\&pid=S1130-527420090002000 01

Bernard, J. M. (2006). Tracing the development of clinical supervision. The Clinical Supervisor, 24(1-2), 3-21. https://doi.org/10 $.1300 / J 001 v 24 \mathrm{n} 01$

Bernard, J. M., \& Goodyear, R. J. (2014). Fundamentals of clinical supervision (5. ${ }^{\mathrm{a}} \mathrm{ed}$.). Boston, MA: Pearson.

Bootzin, R. R., \& Ruggill, J. S. (1988). Training issues in behavior therapy. Journal of Consulting and Clinical Psychology, 56(5), 703-709. Recuperado de https://psycnet.ap a.org/record/1989-09930-001

Callaghan, G. M. (2006). Functional analytic psychotherapy and supervision. International Journal of Behavioral Consultation and Therapy, 2(3), 416-431. ht tps://doi.org/10.1037/h0100794

Caycedo, C. C., Novoa, M. M., \& Ballesteros, B. P. (2008). Análisis de un protocolo de formulación de caso clínico desde las categorías de bienestar psicológico. Universitas Psychologica, 7(1), 231-250. Recuperado de http://www.redalyc.org/arti culo.oa?id $=64770117$

Colegio Colombiano de Psicología. (2009) Deontología y bioética del ejercicio de la psicología en Colombia. Bogotá, Colombia: JAVEGRAF. Recuperado de:
COLPSIC; https://www.colpsic.org.co/quie nes-somos/normatividad

Cruz, J. P. (2009). Enfoque estratégico y formación de terapeutas. Terapia Psicológica, 27(1), 129-142. https://doi.org/10.4067/S0718-480 82009000100013

Ducharme, L. J, Knudsen, H. K., Eु Roman, P. M. (2008). Emotional exhaustion and turnover intention in human service occupations: The protective role of coworker support. Sociological Spectrum, 28, 81-104. https:// doi.org/10.1080/02732170701675268

Ellis, M. V., Berger, L., Hanus, A., Alaya, E. E., Siembor, M. J., \& Swords, B. A. (2014). Inadequate and harmful clinical supervision: Revising the framework and assessing occurrence. The Counseling Psychologist, 42, 434-472. https://doi.org/10 $.1177 / 0011000013508656$

Falender, C. A., \& Shafranske, E. P. (2017). Competency-based clinical supervision: Status, opportunities, tensions, and the future. Australian Psychologist, 52 (2), 86-93. https://doi.org/10.1111/ap.12265

Farber, B. A., \& Hazanov, V. (2014). Informal sources of supervision in clinical training. Journal of Clinical Psychology, 70(11), 1062-1072. https://doi.org/10.1002/jclp.22 127

Follette, W. C., \& Callaghan, G. M. (1995). Do as I do, not as I say: A behavior-analytic approach to supervision. Professional Psychology: Research and Practice, 26(4), 413-421. Recuperado de https://psycnet.ap a.org/buy/1995-41876-001

Gazzola, N., De Stefano, J., Thériault, A., \& Audet, C. (2014). Positive experiences of doctoral-level supervisors-in-training conducting group-format supervision: A qualitative investigation. British Journal of Guidance $\mathcal{E}$ Counselling, 42(1), 26-42. http s://doi.org/10.1080/03069885.2013.799263

Goodyear, R. K., \& Rodolfa, E. (2012). Negotiating the complex ethical terrain of clinical supervision. In S. J. Knapp, M. C. Gottlieb, M. M. Handelsman, \& L. D. VandeCreek (Eds.), APA handbooks in psychology ${ }^{\circledR}$.APA handbook of ethics 
in psychology, Vol. 2. Practice, teaching, and research (pp. 261-275). American Psychological Association. https://doi.org/1 0.1037/13272-013

Guest, P. D., \& Beutler, L. E. (1988). Impact of psychotherapy supervision on therapist orientation and values. Journal of Consulting and Clinical Psychology, 56(5), 653-658. Recuperado de https://psycnet.apa.org/reco $\mathrm{rd} / 1989-09955-001$

Hayes, S. C. (2004). Acceptance and commitment therapy, relational frame theory, and the third wave of behavioral and cognitive therapies. Behavior Therapy, 35, 639-665. Recuperado de https:/www.reachcambridge.com/wp-c ontent/uploads/providing-a-rationale-on-d ifferent-treatments.pdf

Hernández, A. (2007). Supervisión de psicoterapeutas sistémicos: un crisol para devenir instrumentos de cambio. Diversitas. Perspectivas en Psicología, 3(2), 227-238. Recuperado de http://pepsic.bvsalud.org/scielo.php?scri $\mathrm{pt}=$ sci_arttext\&pid $=$ S1794-99982007000 200005

Johnston, L. H., \& Milne, D. L. (2012). How do supervisee's learn during supervision? A grounded theory study of the perceived developmental process. The Cognitive Behaviour Therapist, 5, 1-23. https://doi.org /10.1017/S1754470X12000013

Ladany, N. (2014). The ingredients of supervisor failure. Journal of Clinical Psychology, 70, 1094-1103. https://doi.org/10.1002/jclp.22 130

Milne, D. (2018). Evidence-based clinical supervision: Principles and practice. Leicester, UK: Malden Blackwell Publishing.

Moreira, V., Sabola, A., Beco, L., \& Soares, S. (1995). Psicoterapia fenomenológicoexistencial: aspectos teóricos de la práctica clínica con base en las competencias. Psyke, 4(2), 122-129. Recuperado de http://www.p sykhe.cl/index.php/psykhe/article/view/77

Muñoz-Martínez, A. M., \& Novoa-Gómez, M. M. (2011). Confiabilidad y validación de un modelo de formulación clínica conductual. UniversitasPsychologica, 10(2), 501-519. Recuperado de https://psycnet.ap a.org/record/2012-03247-014

Muñoz-Martínez, A. M., \& Novoa-Gómez, M. M. (2012). Motivos de consulta e hipótesis clínicas explicativas. Terapia Psicológica, 30, 25-36. https://doi.org/10.4067/S0718-4808 2012000100003

Newman, C. F. (2013). Training cognitive behavioral therapy supervisors: Didactics, simulated practice, and "meta-supervision". Journal of Cognitive Psychotherapy, 27(1),5-18. https://doi.org/10.1891/0889-8 391.27.1.5

Novoa-Gómez, M., \& Córdoba-Salgado, O. (2019). Pertinencia de la formación para la supervisión clínica. En G. A. Campos, M. A. Castaño, M. Gaitán \& V. Sánchez (Comps.), Diálogos sobre investigación: avances científicos (pp. 83-116). Bogotá: Konrad Lorenz Editores.

Novoa-Gómez, M., Córdoba-Salgado, O., Rojas, N., Sosa, L., Cifuentes, D., \& Robayo, S. (2019). A descriptive analysis of the interactions during clinical supervision. Frontiers in Psychology, 10, 669. https://doi.o rg/10.3389/fpsyg.2019.00669

Nel, P. W., Pezzolesi, C., \& Stott, D. J. (2012). How did we learn best? A retrospective survey of clinical psychology training in the United Kingdom. Journal of Clinical Psychology, 68, 1058-1073. https://doi.org/1 $0.1002 / j c l p .21882$

O'Donovan, A., Halford, W. K., \& Walters, B. (2011). Towards best practice supervision of clinical psychology trainees. Australian Psychologist, 46,101-112. https://doi.org/10. 1111/j.1742-9544.2011.00033.x

Pulido, D., \& Novoa-Gómez, M. (2014). Clinical case formulation in a context of health. Universitas Psychologica, 13(1), 187-205. https://doi.org/10.11144/Javerian a.UPSY13-1.ccfh

Rodolfa, E., Greenberg, S., Hunsley, J., SmithZoeller, M., Cox, D., Sammons, M., ... Spivak, H. (2013). A competency model for the practice of psychology. Training and 
Education in Professional Psychology, 7(2), 71-83. https://doi.org/10.1037/a0032415

Salgado, A. C. (2007). Investigación cualitativa: diseños, evaluación del rigor metodológico y retos. LIBERABIT, 13(13), 71-78. Recuperado de http://www.scielo.org.pe/pd f/liber/v13n13/a09v13n13.pdf

Shaw, E. (2013). Mentoring or monitoring: Formulating a balance in systemic supervision.Australian $\mathbb{E}$ New Zealand Journal of Family Therapy, 34, 296-310. http s://doi.org/10.1002/anzf.1034

Siddiqui, N. I. (2012). How does a second-wave cognitive behavioural practitioner manage a transition to third-wave practice? Counseling Psychology Review, 27(2), 36-51. Recuperado de https://bps.org.uk/publications/publicati on-by-series/counselling-psychology-revie w/counselling-psychology-review-vol-27-n o-2-june-2012.html

Strauss, A., \& Corbin, J. (2002). Bases de la investigación cualitativa. Técnicas y procedimientos para desarrollar la teoría fundamentada. Medellín: Editorial Universidad de Antioquia.

Taylor, S. J., \& Bogdan, R. (1987). Introducción a los métodos cualitativos de investigación. Barcelona: Paidós.

Watkins, C. E., (2014). The supervisory alliance: A half century of theory, practice, and research in critical perspective. American Journal of Psychotherapy, 68, 19-55. https://doi.org/10.1176/appi.psychot herapy.2014.68.1.19

Weerasekera, P. (2013). The state of psychotherapy supervision: Recommendations for future training. International Review of Psychiatry, 25, 255-264. https://doi.org/10.3109/09540261 .2013 .769431

Notas

* Artículo de investigación. 